\title{
Crystallization of Bisphenol-A Polycarbonate by Using Vapor Transportation Methods
}

\author{
Hiroyuki MochIzUKI, ${ }^{\dagger}$ Toshiko MizoKuRo, Nobutaka TANIGAKI, \\ Takashi HIRAGA, and Norio TANAKA* \\ Photonics Research Institutes, National Institute of Advanced Industrial Science and Technology, \\ 1-8-31 Midorigaoka, Ikeda, Osaka 563-8577, Japan \\ *Dainichi-Seika Color \& Chemical MFG. Co., 1-9-4 Horinouchi, Adachi-ku, \\ Tokyo 123-8555, Japan
}

(Received January 28, 2003; Accepted April 5, 2003)

KEY WORDS Polycarbonate / Crystallization / Vapor / Transportation / Phase Transition /

In recent years, a read-only disk, a write-once optical disk and a rewritable disk have come in popular use. ${ }^{1}$ Especially, the rewritable disk with phase-transition recording consists of a phase-transition layer sandwiched between a polycarbonate substrate and a reflecting layer. In general, the phase-transition layer with an inorganic material such as an alloy of $\mathrm{Ge}-\mathrm{Sb}-\mathrm{Te}$ possesses a mechanism of crystalline-to-amorphous phase transition by heat-mode of laser irradiation. ${ }^{2}$ These optical storage disks were eagerly anticipated to increase storage capacity and to be a simpler structure for cost-down. Furthermore, the phase transition has much potential and is widely applicable for a drive mechanism of organic device as a switching other than the optical storage because it gives us large changes in optical properties. $^{3-5}$

Bisphenol-A polycarbonate (BAPC) is a polymer showing remarkable mechanical properties such as miscibility, transparency, processability, antiweatherability due to its stable amorphous. Thus, it is hard to transform an amorphous structure to a crystalline structure and to crystallize BAPC because of its chain rigidity. Even if BAPC has ability of crystallization, the thermal crystallization of BAPC should be time-consuming. At least, over $200^{\circ} \mathrm{C}, 24 \mathrm{~h}$ is necessary for the first crystalline to develop and one week, or even more, to obtain a crystalline material with a welldeveloped spherulite structure. ${ }^{6-9}$

We have developed a novel method of surface modification termed "vapor-transportation methods".10,11 Simply explaining, this is a method that after a dye is sublimated by heating in a vacuum vessel, the vaporized molecules of dye permeate a polymer from its

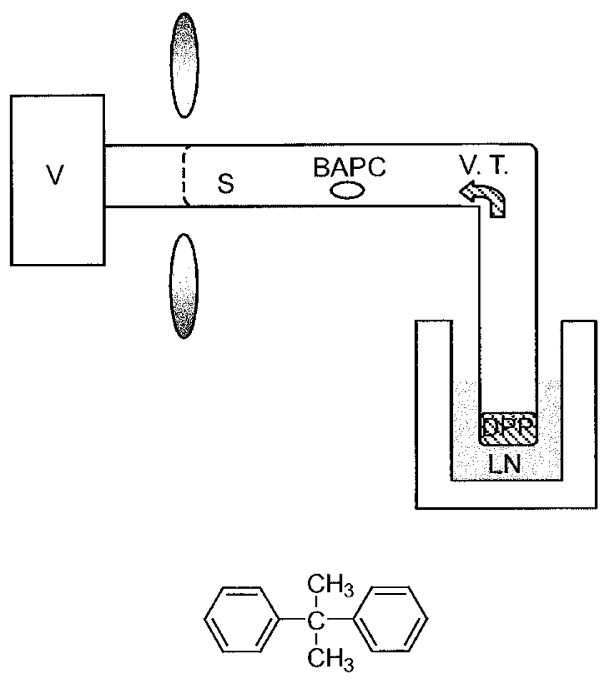

Figure 1. Schematic presentation for the crystallization of BAPC by using "vapor-transportation methods" and a chemical structure of DPP. LN, liquid nitrogen; S, sealing after pumping; $\mathrm{V}$, vacuum pump; V.T., vacuum transportation.

surface in the vessel. Further, this method can control characteristics of polymers without deformation of initial shapes as a post-treatment, namely, color, surface energy, wettability, electrostatic property and so on are tunable.

In this study, we found that BAPC could be crystallized by the vapor-transportation methods and show here that the crystallization behavior of BAPC is controlled by this method. In addition, this method has preferability for applications of BAPC crystallization, which can keep the initial shape after treatment.

Figure 1 shows a schematic presentation for preparation of a sample and the chemical structure of the

${ }^{\dagger}$ To whom correspondence should be addressed (Tel: +81-727-51-9655, Fax: +81-727-51-9637, E-mail: h-mochizuki@aist.go.jp). 


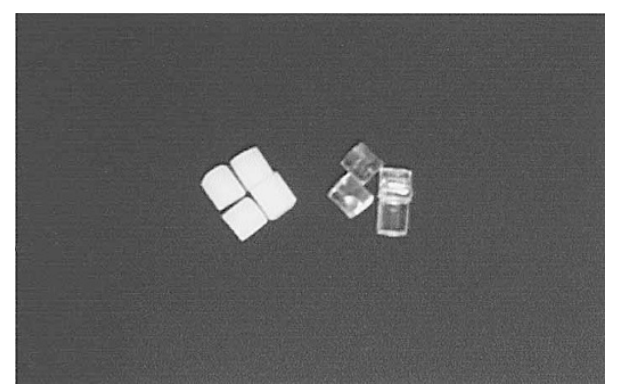

Figure 2. Photograph of the BAPC pellets heat-treated at $150{ }^{\circ} \mathrm{C}$ under reduced pressure (right side) and the BAPC pellets exposed with DPP under the same condition (left side).

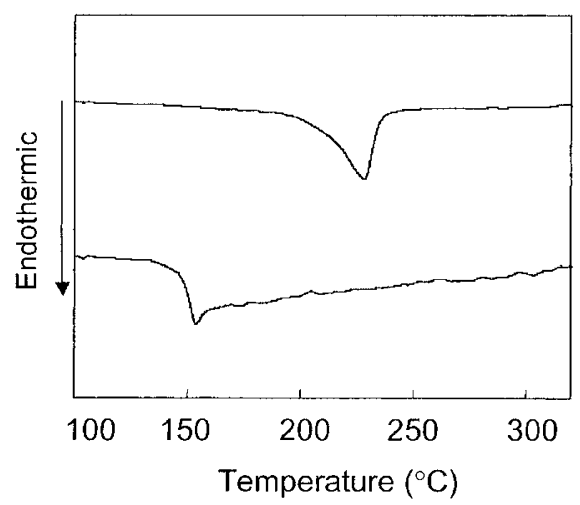

Figure 3. DSC thermograms of the BAPC pellets heat-treated at $150^{\circ} \mathrm{C}$ under reduced pressure (lower side) and the BAPC pellets dispersed with DPP under the same condition (upper side).

compound sublimated in this study. A test pellet of BAPC (purchased from Aldrich Co. LTD; $M_{\mathrm{w}}, 64000$ ) was approximately $\varphi 3 \times 3 \mathrm{~mm}^{3}$ in size and was loaded in a $\varphi 15 \mathrm{~mm}$ glass tube with a sufficient amount of 2,2-diphenylpropane (DPP; obtained from Aldrich Co. LTD) (about few mg). The pumped pressure in the tube was decreased by using a turbo molecular pump (V70; Varian Vacuum Products) as shown in Figure 1. In the pumping, DPP were cooled down by liquid nitrogen in order to reduce the vapor pressure. After an ultimate pressure of around $10^{-6} \mathrm{~Pa}$ was reached, the glass tube was sealed by melting to form an ampoule. The ampoule was set at a constant temperature in an oven, then cooled down slowly over several hours.

Features of BAPC pellets kept at $150^{\circ} \mathrm{C}$ for $24 \mathrm{~h}$ in vacuo and the pellets exposed with DPP under the same condition are shown in Figure 2. As shown in Figure 2, the pellets exposed with DPP were found to turn white, while the pellets kept in vacuo remained unchanged. The whitened BAPC pellets showed the same size and shape as before treatment. Here, in order to analyze structures, each thermodynamic property of these pellets was examined with a differential scanning calorimeter and was shown in Figure 3. In Figure 3 , the unchanged BAPC pellets showed only a glass transition temperature $\left(T_{\mathrm{g}}\right)$ at around $150^{\circ} \mathrm{C}$. On the other hand, whitened BAPC showed only an endothermic peak at $230{ }^{\circ} \mathrm{C}$ due to melting, but did not show any $T_{\mathrm{g}}$. These results suggest that the whitened BAPC pellets are crystallized. After the whitened pellets melted above $240^{\circ} \mathrm{C}$ and were cooled down to room temperature, these pellets changed to be transparent and did not show any melting point but only $T_{\mathrm{g}}$ at around $150^{\circ} \mathrm{C}$ as well as the pellets kept in vacuo or untreated pellets, suggesting that BAPC returned to the amorphous state. Some extent of DPP shows a gas phase at $150^{\circ} \mathrm{C}$ in vacuo and the ampoule becomes packed with the vapor of DPP under the saturated vapor pressure. The vapor of DPP contacts the surface of the BAPC pellets and is considerable to permeate into the BAPC inside, namely, is assumed to be molecularly absorbed in BAPC and to promote the crystallization. It is known that the surface of BAPC is slightly whitened by wiping with a good solvent and reported previously that vapor of a good solvent crystallized BAPC at room temperature in atmospheric pressure. ${ }^{9,12-14}$ However, our study is an original case to indicate the crystallization of BAPC by exposure to a low-molecular weight compound other than the good solvent. Furthermore, If BAPC was exposed with a good solvent like a dichloromethane above room temperature by reported method, an initial shape of BAPC could not be kept.

The degree of crystallinity $\left(X_{\mathrm{c}}\right)$ of BAPC was measured by heat of fusion and calculated by the following equation, $X_{\mathrm{c}}=H / H_{\mathrm{f}} \times 100(\%),{ }^{15}$ where $H$ is a heat of fusion of the sample, $H_{\mathrm{f}}$ is $13.4 \mathrm{cal} \mathrm{g}^{-1}$ according to the reference. The heat of fusion of BAPC prepared in this study was $5.9 \mathrm{cal} \mathrm{g}^{-1}$, so the degree of crystallinity $\left(X_{\mathrm{c}}\right)$ was $44.0 \%$. The crystallinity of BAPC by this method was larger than that by reported method showing $\sim 20 \%{ }^{9}$ The treatment temperature is assumed to affect the crystallinity, because the exposure in this method was carried out at higher temperature than that in reported method.

Table I indicates characteristics of various dopants and crystallization behavior of BAPC dispersed with the dopants. In Table I, crystallization of BAPC was induced by vapor of benzophenone as well as that of DPP. In contrast, BAPC treated with azobenzene in the ampoule did not whitened and not crystallized but changed to clear-light red. At $150^{\circ} \mathrm{C}$ in vacuo, a vapor pressure of azobenzene is considerable to be as much as that of DPP or benzophenone according to the melting point and boiling point of each compound. However, a molecular volume of azobenzene is larger than that of DPP or benzophenone, giving us a prediction that azobenzene molecules absorbed in BAPC prevent crystallization or weak an interaction between BAPC main chains. 
Table I. Characteristics of the dopants and the crystallization of BAPC by dispersion with the dopants

\begin{tabular}{lcccccc}
\hline \multicolumn{1}{c}{ Dopant } & $\mathrm{mp}\left({ }^{\circ} \mathrm{C}\right)$ & $\mathrm{bp}\left({ }^{\circ} \mathrm{C}\right)$ & $\begin{array}{c}\text { Base pressure } \\
(\mathrm{Pa})\end{array}$ & $\begin{array}{c}\text { Treatment } \\
\text { temperature }\left({ }^{\circ} \mathrm{C}\right)\end{array}$ & $\begin{array}{c}\text { Treatment } \\
\text { time }(\mathrm{h})\end{array}$ & Crystallization \\
\hline 2,2-diphenylpropane(DPP) & $29-30$ & $282-283$ & $9.80 \mathrm{E}-06$ & 150 & 48 & $\bigcirc$ \\
Azobenzene & $68-69$ & $293-294$ & $1.33 \mathrm{E}-05$ & 150 & 48 & $\times$ \\
Benzophenone & $48-49$ & $304-306$ & $8.87 \mathrm{E}-06$ & 150 & 48 & $\bigcirc$ \\
\hline
\end{tabular}

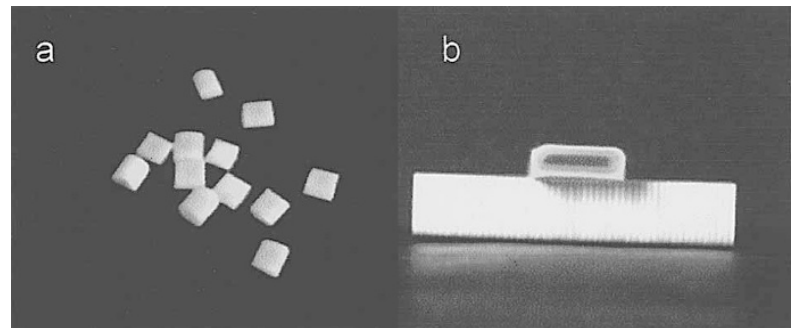

Figure 4. Photographs of the BAPC pellets heat-treated with $4 \mathrm{MNA}$ at $150^{\circ} \mathrm{C}$ under reduced pressure, (a), and the BAPC plate heat-treated under the same condition as described above, (b).

DPP is a colorless compound, so that, a dispersion depth of DPP is beyond observation in crystallized BAPC and is not clear identification of a relation between dispersion and crystallization. In order to explore the relation between dispersion and crystallization, a colored compound needs to be absorbed in BAPC. Therefore, methylnitroaniline derivative like 4methyl-3-nitroaniline (4MNA; obtained from Aldrich Co. LTD; mp, $74-77^{\circ} \mathrm{C}$ ) was selected as a colored compound. In general, colored compounds have larger molecular weights with $\pi$-conjugation. However, among the colored compounds, few possess lower molecular weights than azobenzene. Methylnitoroaniline derivatives, whose molecular weights and molecular volumes are less than azobenzene, show yellow due to intramolecular charge complexes.

Figure 4a shows features of the BAPC pellets doped with $4 \mathrm{MNA}$ at $150^{\circ} \mathrm{C}$ for $24 \mathrm{~h}$. It was found that yellow-whitened BAPC pellets were obtained by doping with $4 \mathrm{MNA}$. Figure $4 \mathrm{~b}$ demonstrates a vertical sectional view of the BAPC plate doped with 4MNA. It is seen that the BAPC plate shows an interface between yellow-whitened region dispersed with 4MNA and undoped transparent one, suggesting that only doped region is crystallized.

As mentioned above, it is reported previously that vapor of good solvent crystallized BAPC at room temperature under atmospheric pressure. ${ }^{8,9}$ Discussion of these reports for the crystallization is that amorphous of BAPC is swollen by the solvent and crystallites play the role of crosslinker as a physical bridge between polymer main chains. On the other hand, some studies reported that the crystallization of BAPC is strongly related to a mobility of main chain and increase in the mobility is believed to promote both nucleation and spherulite growth. ${ }^{16}$ Regarding these results in this study, crystallization of PABC is seemed to be closely related to molecular volumes of the dopants, thus, the mechanism to explain the results and phenomena is considerable to hold for the hypotheses in these papers, especially, the latter assumption. Namely, DPP is considerable to promote the mobility of the main chain of BAPC but azobenzene with larger molecular volume is believed to disturb the mobility.

In conclusion, crystallization of BAPC was performed by vapor-transportation methods. The results in this study lead us to clarify the crystallization mechanisms that a low-molecular weight compound like DPP disperses in BAPC and increases the mobility of polymer main chain, promoting both nucleation and spherulite growth. Further, yellow crystallized BAPC could be obtained by using this method with 4MNA. This method enables us to disperse the functional compounds into the crystallized BAPC. By this method, BAPC was crystallized with benzophenone and methylnitroaniline. Benzophenone shows room-temperature phosphorescence and methylnitroaniline is a nonlinear optical material. Novel optical phenomena would be discovered in crystalline phase by detailed experiment. We are planning to investigate the close crystallization behavior and control the crystallization, further, develop into application of various organic devices.

\section{REFERENCES}

1. S. Ohara, T. Ishida, C. Ichinose, T. Furutani, K. Ishibashi, A. Kurahashi, and T. Yoshida, Proc. SPIE 1991, 187 (1991).

2. N. Ymamada, E. Ohno, K. Nishiuchi, N. Akahira, and M. Takao, J. Appl. Phys., 69, 2849 (1991).

3. G. Attard and G. Williams, Nature, 326, 544 (1987).

4. V. P. Shibaev, S. G. Kostromin, N. A. Plate, S. A. Ivanov, V. Y. Vetrov, and I. A. Yakovlev, Polym. Commun., 24, 364 (1983).

5. H. J. Coles and R. Simon, Polymer, 26, 1801 (1985).

6. B. V. Falkai and W. Rellensmann, Makromol. Chem., 75, 112 (1964).

7. G. Peilstocker, Brit. Plastics, 365 (1962).

8. F. P. Kambour, F. E. Karaz, and J. H. Daenna, J. Polym. Sci., A-2, 4, 327 (1966).

9. J. P. Mercier, G. Groeninckx, and M. Lesne, J. Polym. Sci., C, 16, 2059 (1967).

10. T. Hiraga, Y. Yamasaki, N. Tanaka. K. Hayamizu, and T. Moriya, Chem. Lett., 1791 (1993).

11. T. Hiraga, G. Chen, K. Tsujita, N. Tanaka, Q. Chen, and T. 
Moriya, Mol. Cryst. Liq. Cryst., 344, 211 (2000).

12. F. Gallez, R. Legras, and J. P. Mercier, Polym. Eng. Sci., 16, 276 (1976).

13. R. J. Young and P. Lovell, "Introduction to Polymers", Chapman and Hall Ltd, 1991, chapt. 3, p 137.

14. G. A. Adam, J. N. Hay, I. W. Parsons, and R. N. Haward,
Polymer, 17, 51 (1976).

15. J. M. Jonza and R. S. Porter, J. Polym. Sci., Part B: Polym. Phys., 24, 2459 (1986).

16. A. Conix and L. Jeurissen, "Plasticizers and Plasticization Processes", Advanced Chemistry Series, Symposium, Philadelphia, 1964. 